\title{
ANÁLISIS DE LA SEGURIDAD ACTIVA EN AUTOBUSES MEDIANTE LA REALIZACIÓN DE ENSAYOS VIRTUALES
}

\author{
Daniel Álvarez Mántaras \\ Profesor Titular, Universidad de Oviedo, España \\ Pablo Luque Rodríguez \\ Profesor Titular, Universidad de Oviedo, España \\ Marta Alonso Villarmarzo \\ Ingeniero Industrial, Universidad de Oviedo, España \\ Javier García de Jalón \\ Catedrático, Universidad Politécnica de Madrid, España \\ Alfonso Callejo Goena \\ Doctor Ingeniero Industrial, Universidad Politécnica de Madrid, España
}

\section{RESUMEN}

La seguridad activa de los vehículos se ve afectada por las variaciones de las condiciones de operación y por la degradación de los diferentes sistemas (suspensión, dirección, neumáticos, etc.).

Una de la variables de operación que más afecta a la seguridad es la carga y su distribución el en vehículo. En el caso de los vehículos para el transporte colectivo de personas, por razones obvias, las condiciones de carga son muy variable al igual que la distribución de la misma.

Este trabajo evalúa la sensibilidad de la respuesta dinámica de este tipo de vehículos, con ello de la seguridad activa, ante diferentes condiciones de operación. Para ello, mediante la utilización de un modelo virtual de autobús, desarrollado en el software de simulación dinámica multicuerpo MSC Admas ${ }^{\circledR}$, se simularán los ensayos dinámicos propuestos en por la normativa ISO. Se analiza la respuesta del vehículo ante diferentes condiciones operativas (estados de carga, velocidad, etc). En base a estos análisis se identifica la sensibilidad de las condiciones de seguridad, sirviendo de base para proponer soluciones que permitan minimizar o identificar o predecir las situaciones de riesgo en la circulación de este tipo de vehículos.

\section{INTRODUCCIÓN}

Se entiende por seguridad activa todas aquellas condiciones, sistemas, elementos y factores que tienen por objeto evitar que ocurran los accidentes de tráfico. Se puede afirmar que en 
la circulación de un vehículo existe un nivel de seguridad activa disponible que es función del conductor (capacidades físicas, psíquicas,...) y del vehículo (Luque y Mántaras, 2007).

Cuando el vehículo se pone en movimiento y circula está sometido a la interacción con el entorno, la vía, otros vehículos, peatones,... que generan una "necesidad” de seguridad activa. Se puede afirmar que un vehículo, en cada instante, dispone un nivel de seguridad activa que será función de varios factores (condiciones operativas, el entorno, estado del vehículo, etc). La distancia o separación entre los niveles de seguridad activa disponible y requerida se pueden denotar como "margen de seguridad activa", según se representa gráficamente en la Figura 1.

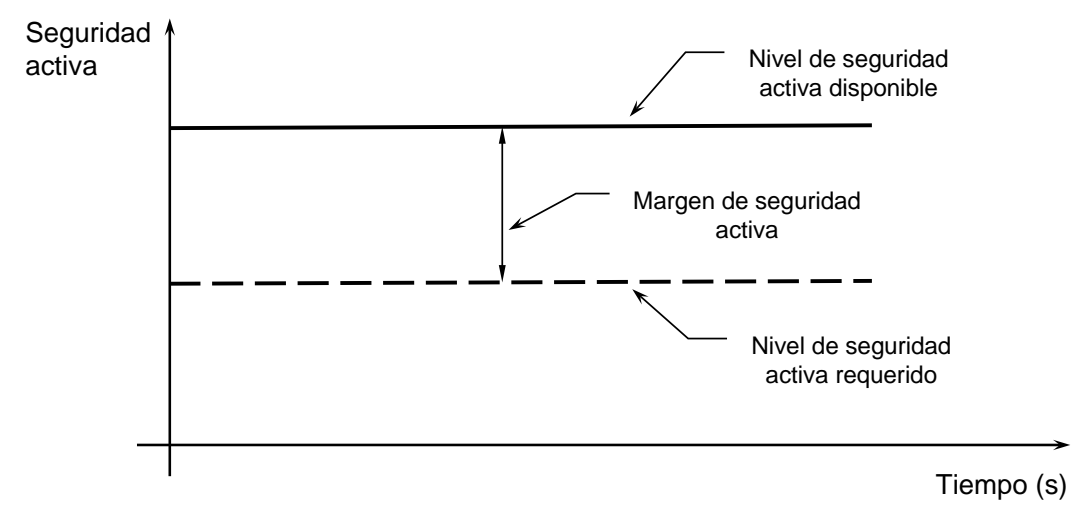

Figura 1 - Seguridad activa disponible y requerida - Margen de seguridad

Siempre que el nivel de seguridad activa disponible sea superior al requerido, la circulación se realizará sin problemas (accidentes o incidentes). Este margen de seguridad es real y por tanto una variable objetiva, que no hay que confundir con los niveles subjetivos de seguridad que puede percibir o declarar un conductor.

El nivel de seguridad activa de un vehículo, del tipo que se analiza en este trabajo, viene limitado por la interacción del neumático con la carretera, es decir, por las fuerzas máximas que es capaz de generar dicha interacción. Estas fuerzas máximas dependen de varios factores (Pacejka, 2002) como la carga vertical, en ángulo de inclinación, el estado de la superficie de contacto, la velocidad, la carga combinada, etc. Por tanto, los límites del vehículo van a ser función del propio vehículo, de las condiciones operativas y de circulación y del entorno.

Aunque, como se ha indicado, el margen de seguridad es una variable objetiva estos niveles no tienen por qué ser iguales para todos los conductores. Es decir, en función de multitud de factores relacionados con el factor humano, no todos los conductores, a igualdad de vehículo y condiciones de circulación (entorno, tráfico, velocidad,...), circulan con el mismo margen de seguridad.

Esa variación, condicionada por factores humanos, se ve afectada con las variaciones en el 
comportamiento del vehículo y, en este tipo de vehículos, puede suceder con la variación en el grado de ocupación. Como se ha indicado, la carga vertical sobre el neumático es una variable que afecta al comportamiento del mismo y, por tanto, a los límites de seguridad del vehículo. En los autobuses, por sus características operativas, la circulación se produce con estados de carga muy variables, no solo en el total de la masa si no, y más importante, en la distribución de la misma. Estas diferentes condiciones operativas afectan a los límites de seguridad activa del vehículo afectando, también, a su respuesta dinámica. Esta variación supone que los conductores la perciban (Malte Rothhämel, 2013), afectando significativamente al proceso de conducción (incremento del stress de conducción) y reduciendo, con ello, ese margen de seguridad.

La finalidad que persigue el presente trabajo es analizar la seguridad activa en autobuses evaluando cómo puede afectar el número de pasajeros y su colocación mediante la realización de ensayos virtuales a través del software de simulación dinámica multicuerpo Adams/View de la compañía MSC Software. Drugge y Magnus (2014) utilizan este software para la evaluación del comportamiento de este tipo de vehículos ante un viento lateral. Prado (2001) evalúa la dinámica de un autobús mediante ensayos virtuales realizados con Adams.

\section{METODOLOGÍA}

\subsection{Modelo virtual}

Para la evaluación de la seguridad se utiliza el modelo multicuerpo de autobús que se muestra en la Figura 2. El modelo virtual, de 29 grados de libertad, reproduce un autobús de 45 plazas (más 3 de tripulación) con suspensión neumática de tipo paralelogramo delantero y eje rígido multibrazo trasera.

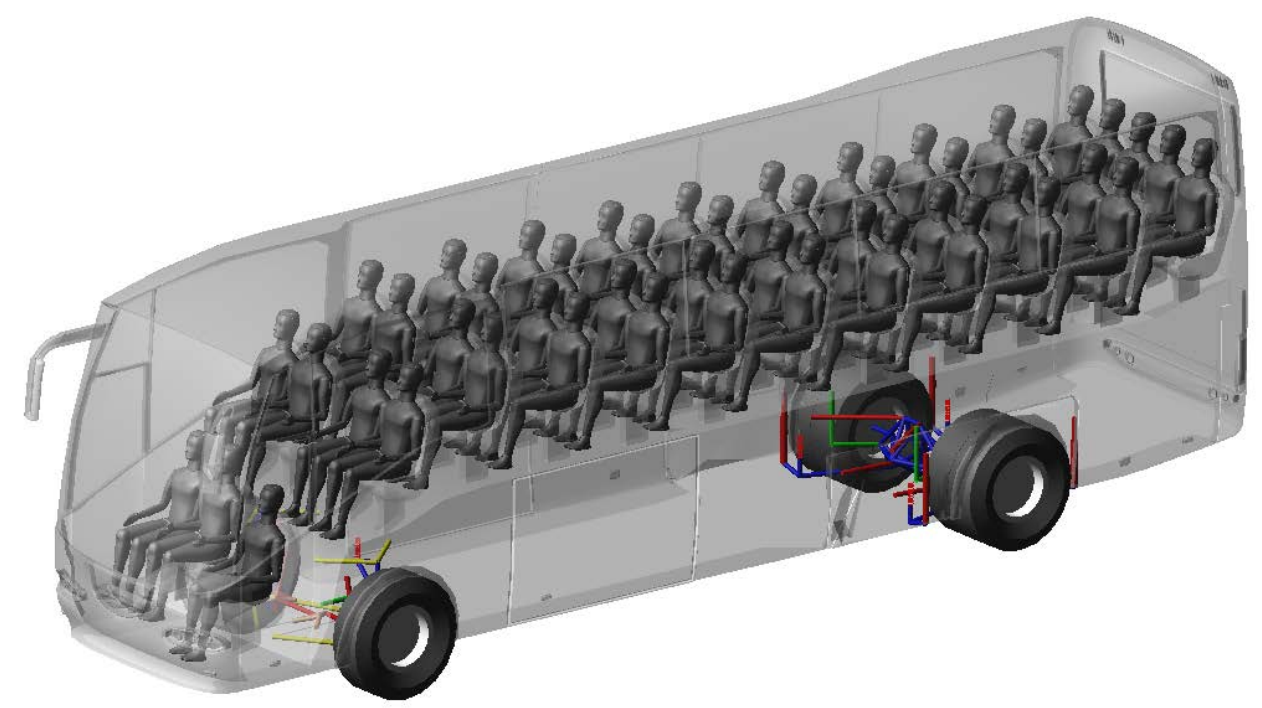

Figura 2 - Modelo virtual utilizado para los ensayos con carga completa

Las entradas al sistema son la velocidad de circulación y el giro de volante. Se implementa 
un control PID para regular el par en la ruedas del eje trasero en función de la velocidad de consigna. De esta manera se reproducen las acciones del conductor sobre el vehículo de la misma manera que el sistema real.

Sobre este modelo se desarrollan cuatro variantes que representan, a su vez, cuatro casos de carga: orden de marcha, media carga con distribución delantera, media carga con distribución trasera y carga completa.

\begin{tabular}{|l|c|c|c|}
\hline \multicolumn{1}{|c|}{ Estado de carga } & Masa $(\mathrm{kg})$ & Reparto delantero/trasero & Altura c.d.g. (m) \\
\hline Orden de marcha & 13542 & $33 \% / 67 \%$ & 1,08 \\
\hline Media carga delantera & 15112 & $38 \% / 62 \%$ & 1,2 \\
\hline Media carga trasera & 15317 & $30 \% / 70 \%$ & 1,2 \\
\hline Carga completa & 17887 & $35 \% / 65 \%$ & 1,3 \\
\hline
\end{tabular}

Tabla 1 - Estados de carga analizados

\subsection{Ensayos}

El modelo virtual es ensayado siguiendo los procedimientos definidos, para ensayos reales, por las normativas ISO 11012, 11026, 14792 y 14793 aplicables a camiones y tráileres con una masa máxima por encima de 3,5 toneladas y autobuses y autobuses articulados con una masa máxima por encima de 5 toneladas, de acuerdo a las categorías de vehículos, ECE y EC, M3, N2, N3, O3 y O4).

La norma ISO 11012 define dos ensayos de lazo abierto para determinar las características del "On-Centre Handling” de un vehículo, en respuesta a entradas de dirección específicas: Weave Test y Transitional Test. La norma ISO 11026 define un ensayo en lazo abierto para determinar la estabilidad en balanceo de un vehículo sobre una curva con superficie seca. La norma ISO 14792 define ensayos para determinar la respuesta direccional en estado estacionario de vehículos pesados. La norma ISO 14793 define los métodos de prueba para determinar la respuesta transitoria de vehículos. En todas las normas se definen las variables a medir, así como los métricos y gráficos a representar.

Los cuatro casos de carga son simulados siguiendo lo recogido en la normativa descrita para las velocidades de $80 \mathrm{~km} / \mathrm{h}, 90 \mathrm{~km} / \mathrm{h}$ y $100 \mathrm{~km} / \mathrm{h}$. Se trata de analizar la respuesta a la velocidad máxima permitida para estos vehículos en autopistas, autovías y resto de las vías fuera de poblado (Reglamento General de Circulación, 2015)

\section{RESULTADOS}

Tras la realización de los diferentes ensayos se constata la variación del comportamientos dinámico del vehículo. Se analizan, a continuación, las variables más significativas para los diferentes ensayos realizados. 
A partir del ensayo ISO 11012 Weave Test (giro en alternativo), en base al ciclo de histéresis obtenido representando la velocidad de guiñada respecto al giro volante, se obtienen las zonas muertas para cada configuración que se muestran en la Figura 3.

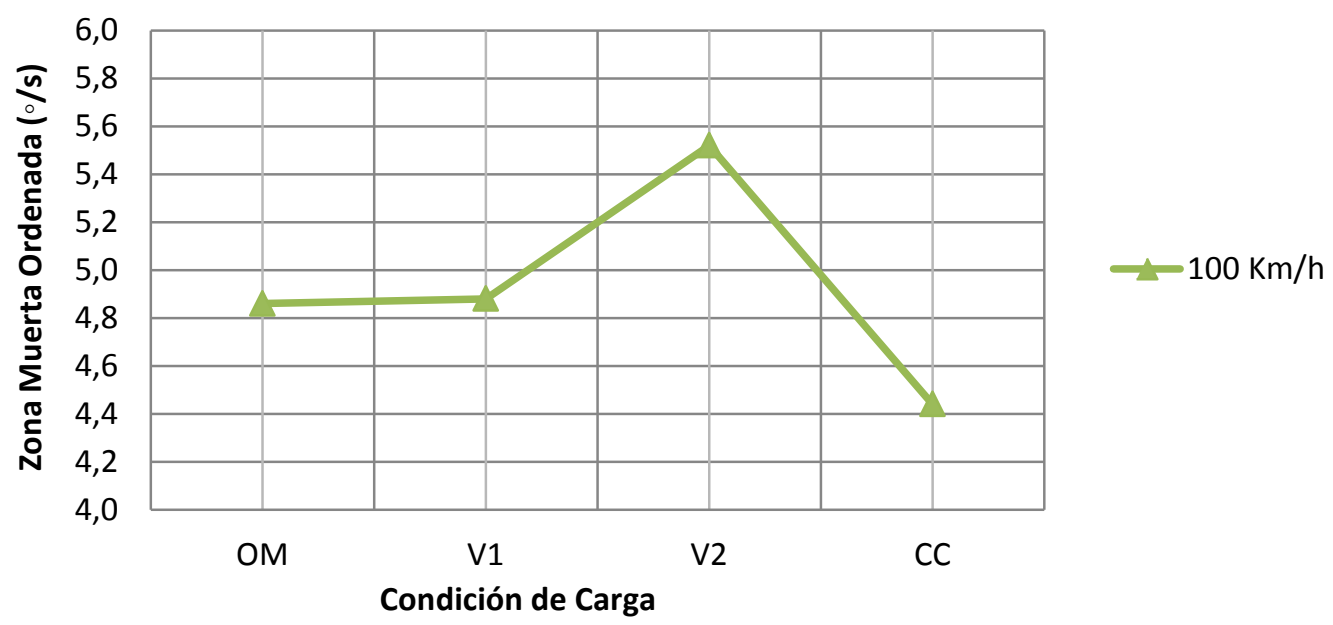

Figura 3 - Zona muerta en guiñada en función de la condición de carga (OM orden de marcha, V1 media carga delantera, V2 media carga trasera, CC carga completa)

En la Figura 4 se muestra la zona muerta en base al ciclo de histéresis de la aceleración lateral respecto al giro de volante.

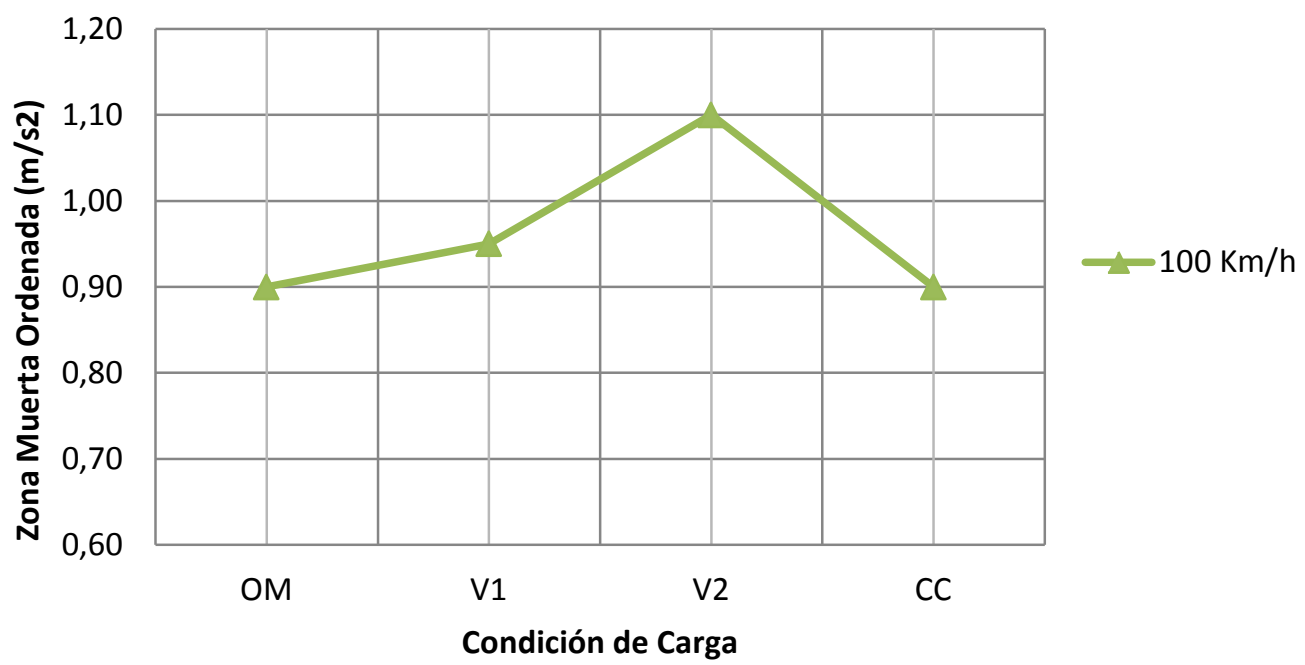

Figura 4 - Zona muerta en aceleración en función de la condición de carga

Se observa como la condición de media carga con disposición trasera supone una variación significativa de estos dos métricos, del 12 \% para la velocidad de guiñada y del 22\% para la aceleración. Estas zonas muertas están relacionadas con la percepción del conductor de la respuesta dinámica del vehículo en circulación en recta (Norman, 1984; Farrer, 1993), por tanto, los porcentajes indicados son lo suficientemente significativos como para afectar a esa percepción. 
Analizando el tiempo de respuesta de la velocidad de guiñada ante la entrada de volante (Figura 5) se observa que el vehículo en orden de marcha o con la carga en la parte trasera es entre un 15 y $20 \%$ más lento que las otras dos configuraciones.

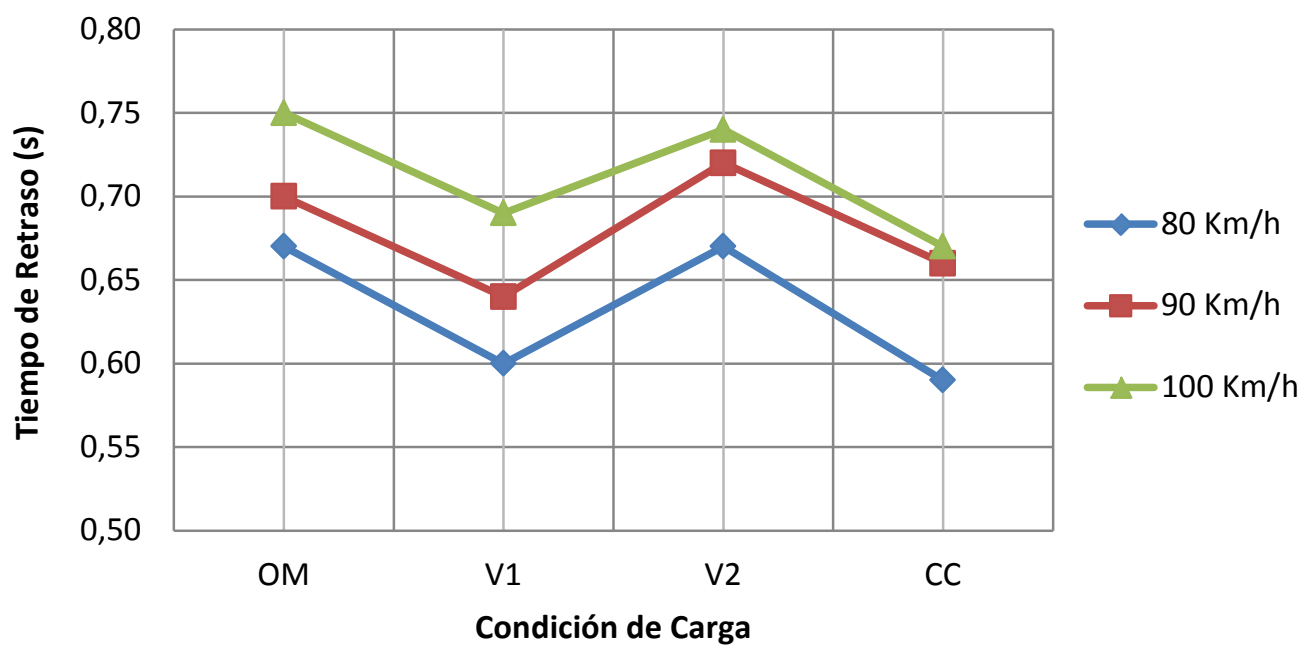

Figura 5 - Tiempo de retraso de la velocidad de guiñada

Cuando se analiza la respuesta transitoria, ISO 11012 test de transición, no se observan variaciones significativas para velocidades bajas. Sin embargo, observando el gradiente de aceleración lateral (Figura 6) a velocidades elevadas, las variaciones respecto al vehículo en orden de marcha llegan al $10 \%$.

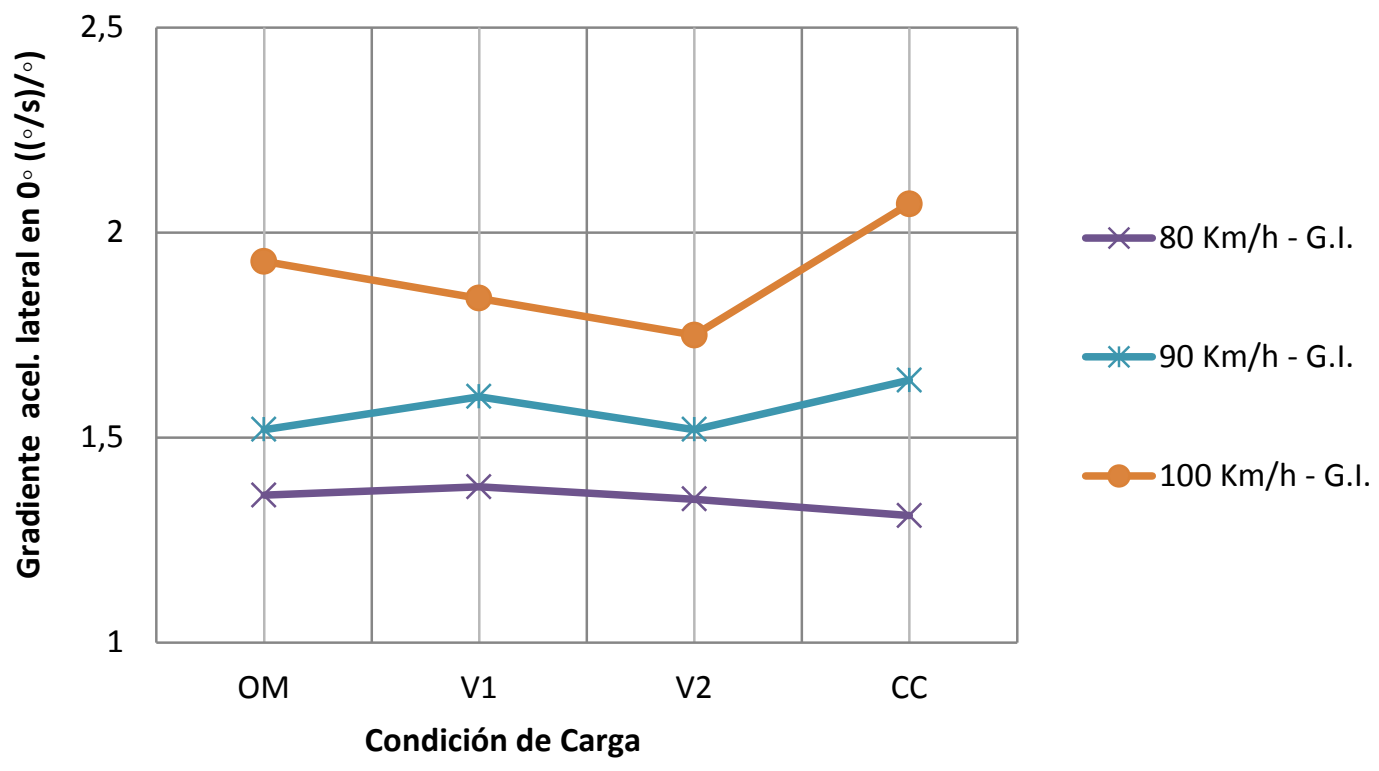

Figura 6 -Gradiente de la aceleración lateral en función de la condición de carga

Cuando se analiza la estabilidad en curva de las cuatro configuraciones, según ensayo ISO 11026, se observa cómo la aceleración límite de vuelco se reduce con la carga, de tal forma 
que en carga completa es un 19\% menor que en orden de marcha. Esto se debe, fundamentalmente, a la elevación del centro de gravedad del vehículo con el grado de ocupación.

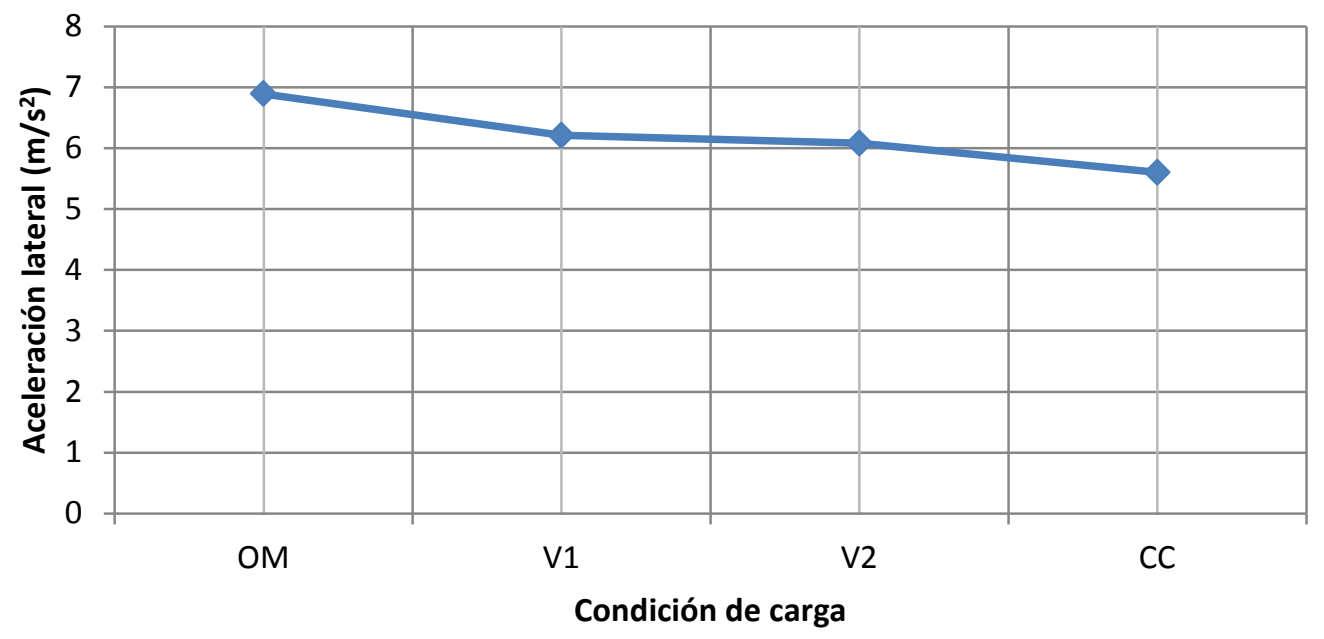

Figura 7 - Aceleración límite de vuelco en función de la condición de carga

La carga también influye en coeficiente de balanceo del vehículo (Figura 8). Este coeficiente representa el ángulo de balanceo de la carrocería para una aceleración lateral equivalente a 1 g. Esa rotación de la caja respecto al eje longitudinal (balanceo) es percibida por el conductor y ocupantes en el paso por curva siendo, como se ve en la Figura 8, un 90\% mayor en carga completa que en orden de marcha.

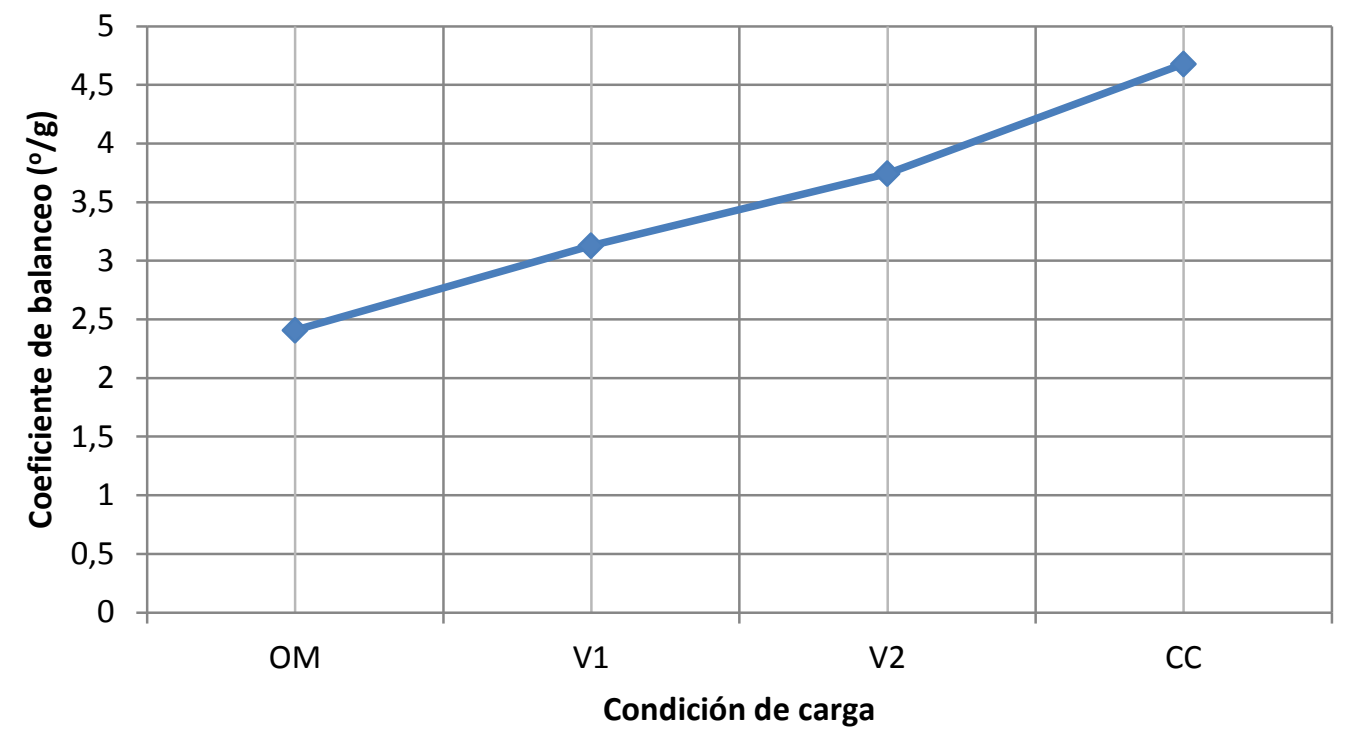

Figura 8 - Coeficiente de balanceo en función de la condición de carga

En un paso por curva cuasi estático (ISO 14792) el comportamiento direccional (subvirador/sobrevirador) presenta diferencias entre las cuatro condiciones de carga (Figura 9). Para una aceleración de paso por curva de $4 \mathrm{~m} / \mathrm{s}^{2}$ las variaciones en el ángulo de deriva del vehículo superan el $12 \%$. 


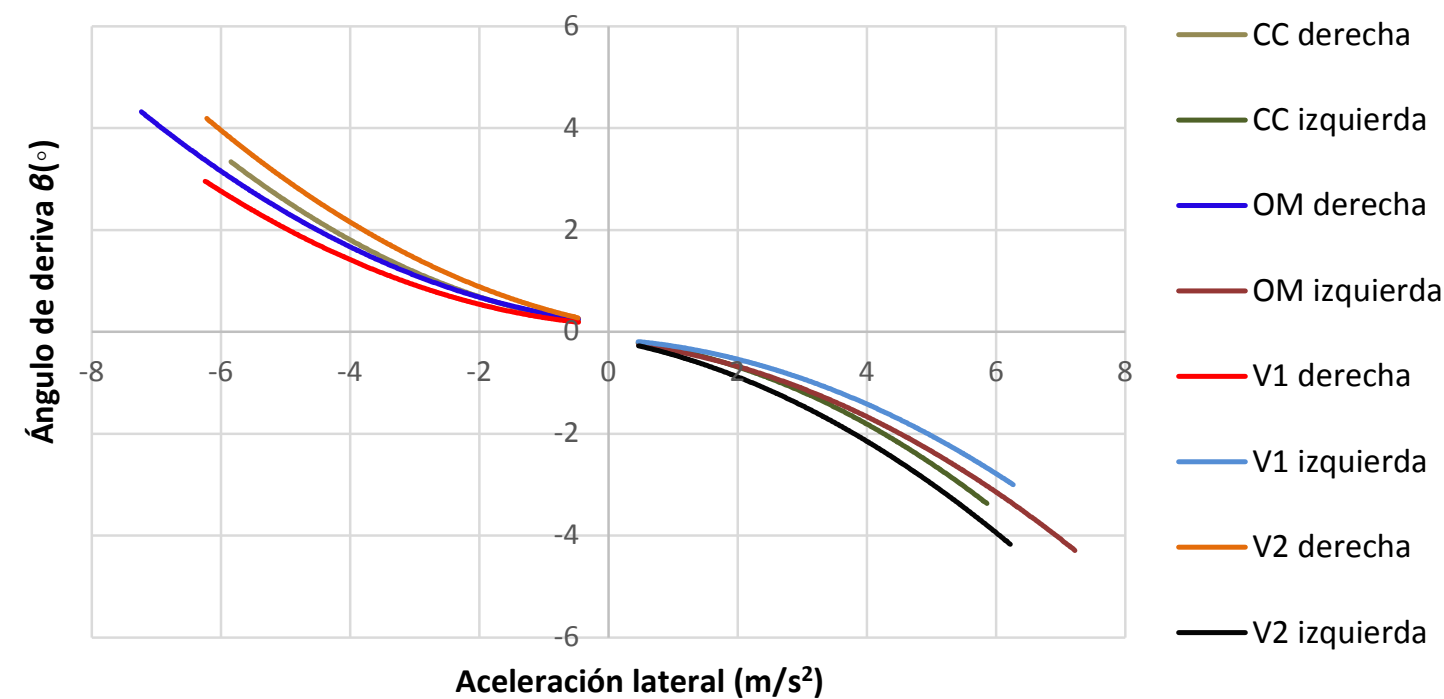

Figura 9 - Aceleración lateral en función del ángulo de deriva del vehículo

Ante el ensayo que evalúa la respuesta transitoria en curva (ISO 14793) se observa una variación significativa (20\%) en el métrico del tiempo de respuesta a la velocidad de guiñada. Esto significa un retraso en la respuesta direccional ante la entrada de volante en curva que afecta significativamente a la percepción del conductor (Masato Abe, 2009).

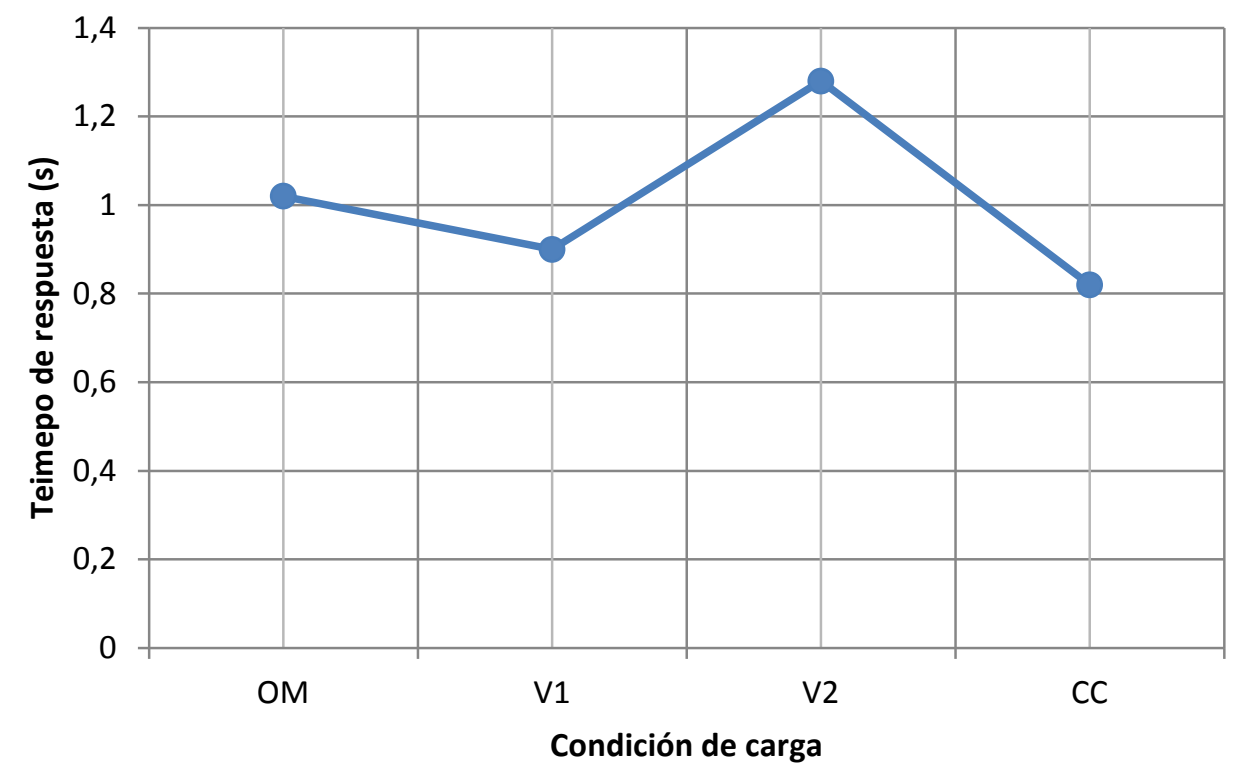

Figura 10 -Tiempo de respuesta de la velocidad de guiñada

\section{CONCLUSIONES}

La aplicación de un modelo virtual de autobús ha permitido constatar la variación del comportamiento dinámico y, con ello, de los límites de seguridad activa con diferentes 
condiciones de carga. Se ha limitado el análisis a tres condiciones de carga, orden de marcha, media carga y carga completa, con dos distribuciones diferentes a media carga. Aunque en la realidad pueden existir más variantes, las tres condiciones de carga han sido suficientes para evidenciar cómo afecta a la dinámica del vehículo.

El número y disposición de los pasajeros de un autobús da lugar a la variación de la posición del centro de gravedad (Tabla 1) y de los momentos de inercia. Con ello, la distribución y cantidad carga sobre los ejes también se ve modificada. Además, el disponer de una suspensión neumática supone que se modifiquen las características elásticas de estos elementos afectando a las transferencias de carga longitudinal (en tracción o frenado) y transversal (en paso por curva). Por tanto, los neumáticos, en cada una de las variantes, trabajaran en diferentes condiciones de carga vertical y de posición. Esto supone que su respuesta y sus límites (Pacejka, 2002) sean diferentes y, con ello, los límites de seguridad activa propios del vehículo.

Los diferentes ensayos realizados han permitido cuantificar variaciones entre el 10 y $30 \%$ de métricos de la respuesta dinámica que están relacionado con la percepción del conductor. Por tanto, afectando a los límites de seguridad activa del conductor.

Como conclusión, en un vehículo como el analizado, la posición de los viajeros afecta a la seguridad por lo que se debería definir la ubicación óptima de los pasajeros cuando no se circula a carga completa. Por otro lado, se constata la necesidad de definir métricos objetivos que permitan cuantificar el margen de seguridad en cada instante. Con ello se podrían definir sistemas de apoyo a la conducción o de evaluación de conductores.

\section{AGRADECIMIENTOS}

La publicación de este este artículo de investigación ha sido posible gracias a la financiación proporcionada por el Ministerio de Economía y Competitividad, a través de los proyectos pertenecientes al Plan Nacional de I+D+i TRA2009-14513-C02 y TRA201238826-C02.

\section{REFERENCIAS}

\section{Libro}

LUQUE RODRÍGUEZ, P. y ÁLVAREZ MÁNTARAS, D. (2007). Investigación de Accidentes de Tráfico. Manual de reconstrucción. Netbiblo. La Coruña.

PACEJKA, B.H. (2002). Tyre and Vehicle Dynamics. Butterworth-Heinemann, Oxford.

Malte Rothhämel (2013). Characterisation and Utilisation of Steering Feel in Heavy Trucks Doctoral Thesis in Vehicle Engineering. Royal Institute of Technology KTH Vehicle Dynamics 
Masato Abe (2009). Vehicle Handling Dynamics, Theory and Application. ButterworthHeinemann. Oxford.

\section{Artículo de revista}

Norman, K. D., Objective Evaluation of On-Center Handling Performance, SAE Paper 840069

Farrer, D. G., An Objective Measurement Technique for the Quantification of On-Centre Handling Quality, SAE Paper 930827

Drugge y Magnus Juhlin (2010) Aerodynamic loads on buses due to crosswind gusts: extended analysis. Vehicle System Dynamics: International Journal of Vehicle Mechanics and Mobility, 48:S1, 287-297, DOI: 10.1080/00423111003739814

Prado, M., Cunha, R., Neto, Á., Costa, A. et al. (2001). Bus Handling Analysis and Experimental Validation Using the Multibody System Technique. SAE Technical Paper 2001-01-3966, 2001, doi:10.4271/2001-01-3966

\section{Series}

ISO 15037-2. Road vehicles - Vehicle dynamics test methods - Part 2: General conditions for heavy vehicles and buses

ISO 11012:2009. Heavy commercial vehicles and buses -- Open-loop test methods for the quantification of on-centre handling -- Weave test and transition test

ISO 11026:2010. Heavy commercial vehicles and buses -- Test method for roll stability -Closing-curve test

ISO 14792:2011. Road vehicles -- Heavy commercial vehicles and buses -- Steady-state circular tests

ISO 14793:2011. Road vehicles -- Heavy commercial vehicles and buses -- Lateral transient response test methods

Reglamento General de Circulación (2015). Última modificación 18 de julio de 2015. Real Decreto 1428/2003, de 21 de noviembre, por el que se aprueba el Reglamento General de Circulación para la aplicación y desarrollo del texto articulado de la Ley sobre tráfico, circulación de vehículos a motor y seguridad vial, aprobado por el Real Decreto Legislativo 339/1990, de 2 de marzo. 